zweiten Hauptsatzes. Im vierten Buche werden zuerst die Gesetze des Schmelzens und Verdampfens untersucht; daran schlieBt sich dann die Lehre von dem thermodynamischen Potential und von seinen Anwendungen auf allgemeinere Fälle von Zustandsänderungen, sowie auf die kritischen Zustände. Das letzte Buch endlich enthält die Lehre von Wärme-Leitung und -Strahlung.

Der Darstellung der physikalischen Erscheinungen selbst habe ich eine Einleitung vorangeschickt. In ihr sind allgemeine Ausführungen über Methode und Gegenstand der physikalischen Forschung, Angaben über Messungen und Maßeinheiten, sowie einige mathematische Sätze aufgenommen worden, von denen gelegentlich Gebrauch zu machen ist. Ich hoffe mit dieser Erinnerung an die Schulbank viclleicht dem einen oder anderen meiner Leser einen Dienst zu erweisen.

Ein ausführliches Sachregister soll an den Schluß des zweiten Bandes gestellt werden.

Die Figuren sind für das vorliegende Buch beinahe sämtlich neu gezeichnet worden; nur wenige Zeichnungen von Apparaten sind aus Katalogen und aus RIEss' Lehre von der Reibungselektrizität kopiert. Die Zeichnungen von Interferenzkurven bei Kristallen sind der physikalischen Kristallographie von LieBISCH entnommen.

Wenn ich an meine eigene Studienzeit zurückdenke, so verweilt meine Erinnerung besonders gern bei den Stunden, in denen ich WILHELM WeBERS Vorlesung über Experimentalphysik hörte. Wer seine elektrodynamischen Maßbestimmungen gelesen hat, kann sich wohl einen Begriff machen von der Kunst, mit der er den Zusammenhang der Erscheinungen zu entwickeln und Schritt für Schritt die Erkenntnis zu erweitern und $\mathrm{zu}$ vertiefen wußte. So gestaltete sich vor allem die von ihm mit Vorliebe behandelte Elektrizitätslehre zu einem Kunstwerke, dessen dramatischen Aufbau von Stunde zu Stunde zu verfolgen mir eine Quelle des reinsten Genusses war. Möchte ein Hauch von diesem Geiste auch in meiner Darstellung zu spüren sein!

November 1895 .

\title{
Eduard Riecke.
}

\section{Vorwort zur fünften Auflage.}

Seit dem Erscheinen der ersten Auflage dieses Buches hat die Physik eine Epoche groBer Entdeckungen erlebt, welche auf unsere physikalischen Anschauungen die tiefste Wirkung ausgeübt haben. Zwar der RöNTGEN-Strahlen konnte in der ersten Auflage noch flüchtig gedacht werden. Die Entdeckung der Becquereu-Strahlen und des ZeEmaNEffektes folgten später; ebenso die fundamentalen Untersuchungen über Kathodenstrahlen und Kanalstrahlen, durch welche über das ganze Gebiet 
der Entladungserscheinungen in verdünnten Gasen neues Licht verbreitet und eine übersichtliche Darstellung dieses Gebietes erst ermöglicht wurde. Auf der anderen Seite entwickelte sich die Lehre von den Gasionen und von der Leitung der Elektrizität in dichten Gasen. Ein neues Gebiet, welches für unsere atomistischen Anschauungen von der gröBten Bedeutung geworden ist, erschloB sich in der Radioaktivität. Der Mrchelsonsche Interferenzversuch gewann ein ganz neues und allgemeines Interesse, als wesentlichste Stütze des sogenannten Relativitätsprinzips. Unsere Vorstellungen von der Konstitution der Materie wurden erweitert durch die Untersuchungen über flüssige Kristalle. Die kinetischen Anschauungen der Wärmelehre fanden eine schöne Bestätigung in den Untersuchungen über die BRownsche Molekularbewegung. Der von NerNst aufgestellte Wärmesatz erwies sich als ein wertvoller Leitfaden zur Aufstellung neuer Beziehungen zwischen den thermischen Eigenschaften der Körper. Die aufeinanderfolgenden Auflagen des Lehrbuches geben von dieser Entwickelung ein anschauliches Bild. Den Erscheinungen der elektrischen Entladung und Leitung in Gasen wurde schon in der zweiten Auflage ein besonderes Buch gewidmet. In der vierten Auflage wurde der Lehre von der Radioaktivität zum ersten Male ein besonderes Kapitel eingeräumt; dieses ist in der fünften Auflage entsprechend den Fortschritten der Wissenschaft einer gründlichen Umarbeitung unterworfen worden. Das gleiche gilt von den Abschnitten, in welchen die Kanalstrahlen behandelt werden. Völlig neu sind in der fünften Auflage die Abschnitte über den Michelson schen Versuch, die flüssigen Kristalle, die Brownsche Molekularbewegung und den Nernstschen Wärmesatz. Aber auch abgesehen von solchen Einschaltungen, welche der Fortschritt der Wissenschaft mit sich gebracht hat, ist der Inhalt des Buches im Vergleich mit der ersten Auflage nach mancherlei Richtungen vermehrt worden. In der zweiten Auflage wurde dem Verhalten starrer Körper in bewegten Flüssigkeiten ein besonderes Kapitel gewidmet; in der dritten erfuhr die geometrische Optik eine wesentliche Erweiterung. Die Theorie der Leistungsfähigkeit des Mikroskops wurde anf Grund der Beugungserscheinungen nach HецмноLтz und AввE entwickelt. Auf kleinere Einschaltungen und Verbesserungen, welche in der fünften Auflage gemacht worden sind, brauche ich nicht einzugehen. Für die Überlassung der Figuren zur GaEDE-Pumpe habe ich der Firma LeYBold in Köln zu danken.

Bei den früheren Auflagen war ich meinen Kollegen Vorgt, Pockels, Wiechert, Simon und Stark für die Durchsicht der Korrekturbogen und für manche wertvolle Bemerkung zu Dank verpflichtet. Derselben Aufgabe haben sich bei der fünften Auflage die Herren Dr. MADELUng, Dr. RüMELIN und Fräulein ANNa Disse unterzogen. Ihnen sage ich auch an dieser Stelle für ihre Bemühungen meinen wärmsten Dank.

Göttingen, 24. Oktober 1911.

Eduard Riecke. 